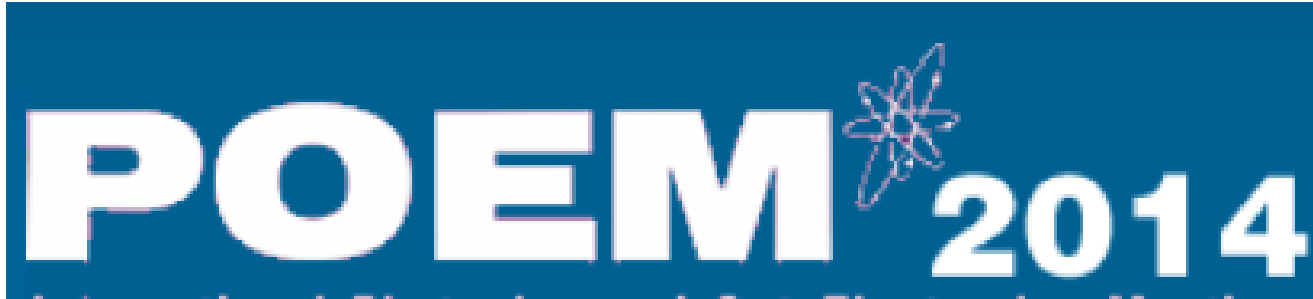

\title{
Ultra-low volume tunable slot photonic crystal cavities for sensing
}

Samuel Serna, Charles Caer, Weiwei Zhang, Xavier Le Roux, and Eric Cassan Institut d'Electronique Fondamentale

University Paris-Sud, FRANCE CNRS UMR 8622

eric.cassan@u-psud.fr 


\section{Slow light in photonic crystal waveguides}

- Strong enhancement of the optical field

- Engineering a flat group index curve can cancel the group velocity dispersion: this is done by tuning the hole lattice

- Losses mainly due to backscattering induced by disorder. Scale with $n_{G}^{2}$

- Slight stretch of the lattice constant at the interface between fast and slow modes ensures high injection efficiency.
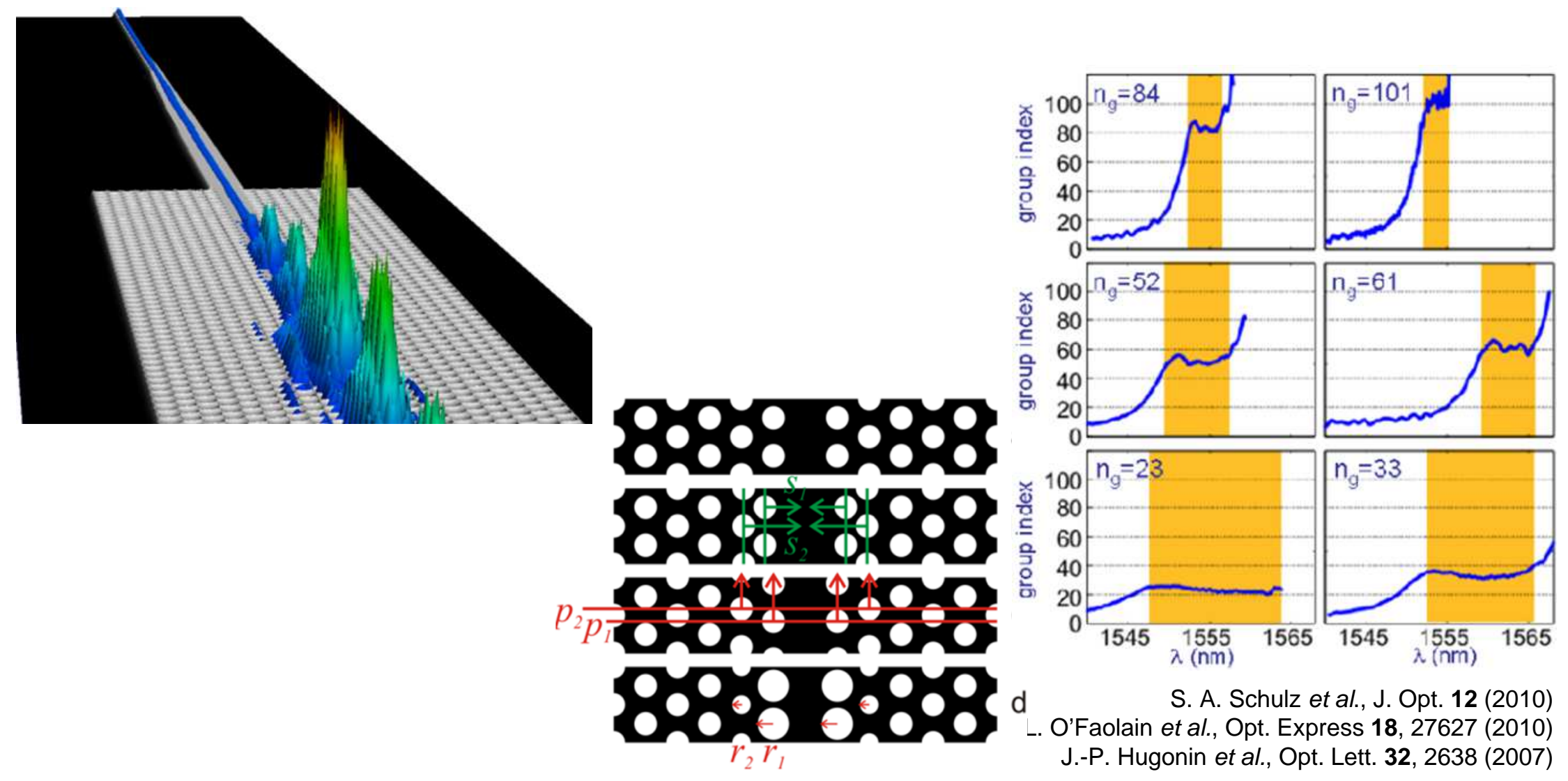


\section{A tool for $X^{(3)}$ nonlinear optics}

- Third Harmonic Generation

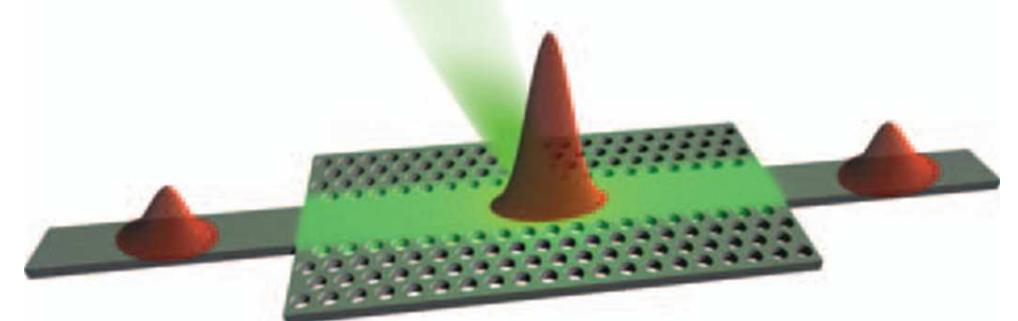

B. Corcoran et al., Nat Photonics 3, 206 (2009)

- Stimulated Raman scattering

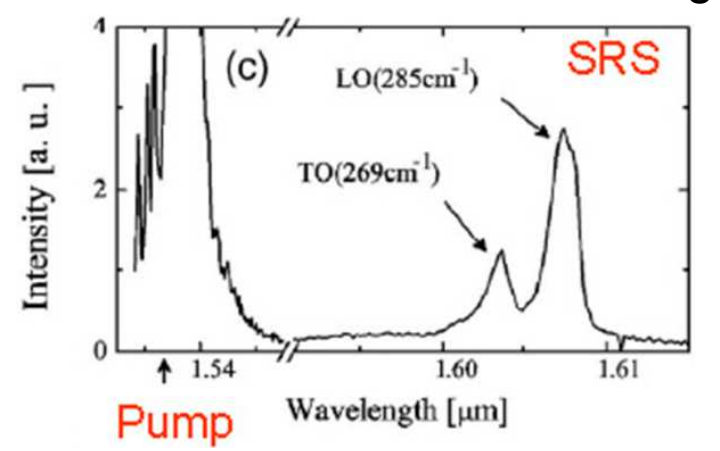

J. F. McMillan et al., Opt. Lett 31, 1235 (2006)
- Deqenerate Four Wave Mixing

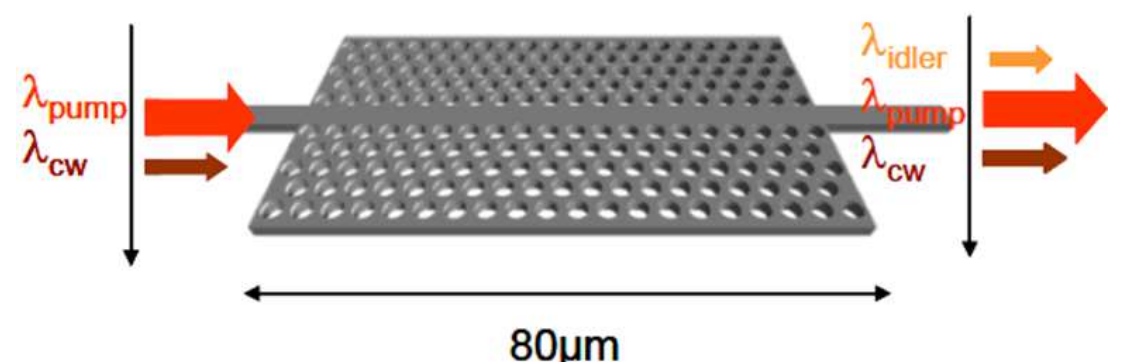

C. Monat et al., Opt. Express 18, 22915 (2010)

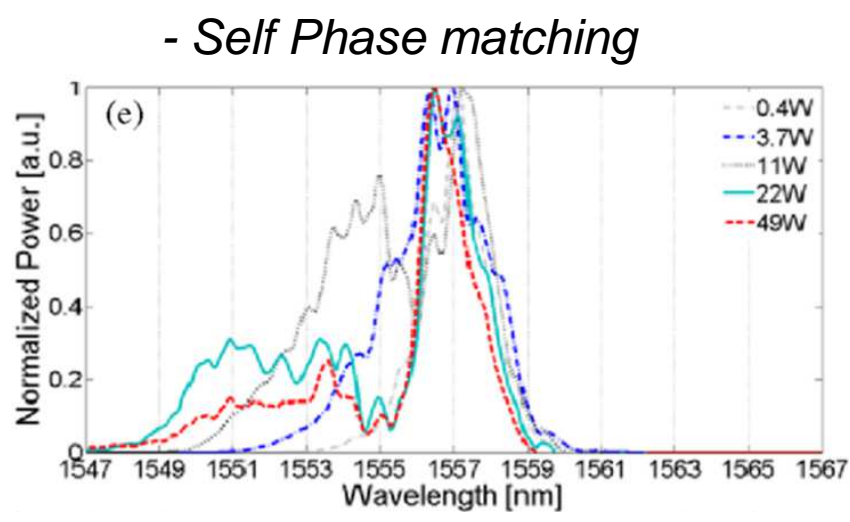

C. Monat et al., Opt. Express 17, 2944 (2009)

However:

- $\quad$ Silicon nonlinearities are limited by Two-Photon Absorption and Free Carrier Absorption

- $\quad$ Silicon is centrosymmetric (no $X^{(2)}$ effects)

- $\quad$ Figure of merit of silicon is relatively low $(<0.9)$ compared to polymers It is therefore preferable to confine the light in a nonlinear cladding layer...

C. Koos et al., Opt. Express 15, 5976 (2007) 


\section{Slot Photonic Crystal Waveguides (SPCW)}

- Combination of two types of confinements:

- $\quad$ Slowed pulse -> increased intensity

- High index-contrast interface in the slot

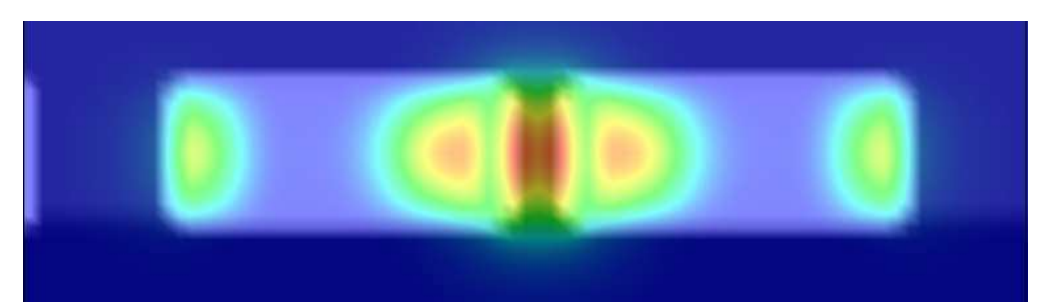

- $\quad$ Two even guided modes depending on both photonic crystal and slot parameters

- $\quad$ One mode comes from the W1 defect line, the other from the slot waveguide

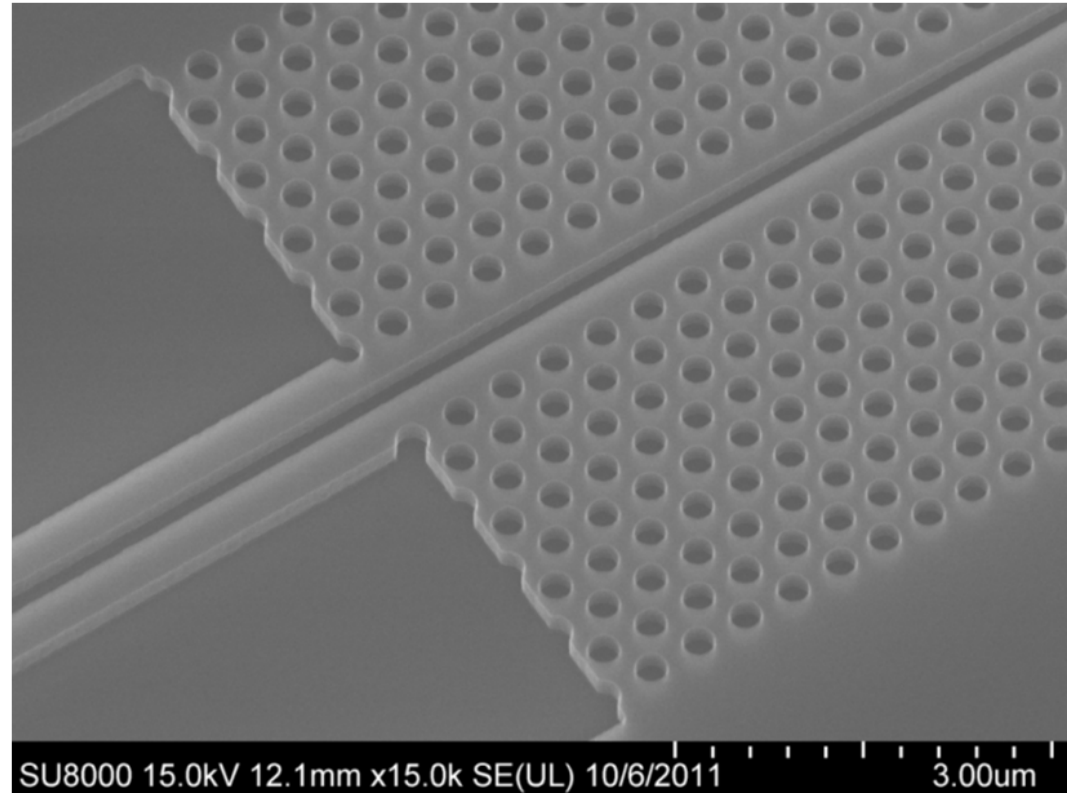

(IEF / CTU-MINERVE)

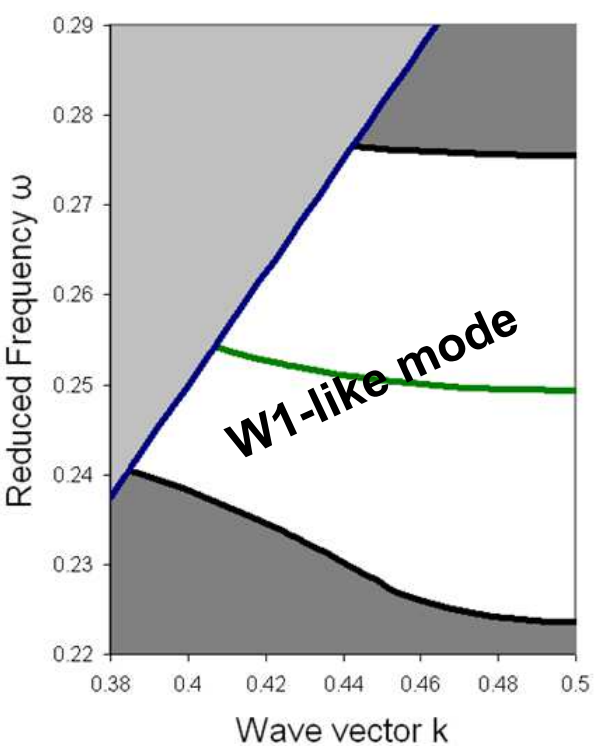

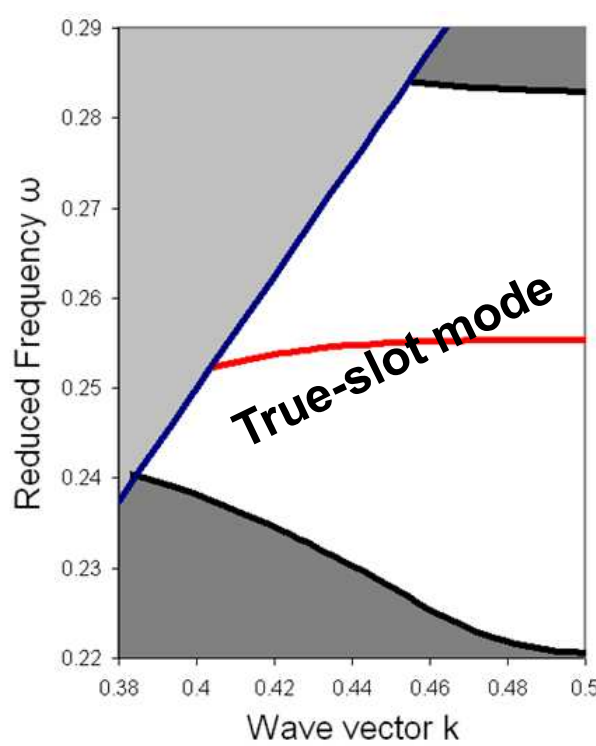

$40 \%$

A. Di Falco et al., Appl. Phys. Lett. 92, 083501 (2008) J. Gao et al., Appl. Phys. Lett. 96, 051123 (2010) 


\section{The extrinsic loss issue}

Losses mainly due to backscattering induced by disorder. Scale with ... $n_{G} \ldots n_{G}^{2} \ldots$

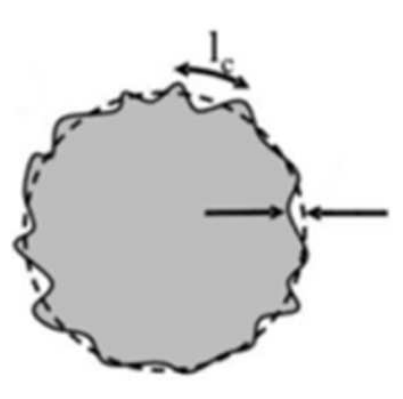

- Reflexions at the strip wg/PhC waveguide $+$

- Out-of-plane mode Bloch coupling above the light line

- Disorder-induced losses

- Backscattering and multiple scattering

- Bandgap edge variations
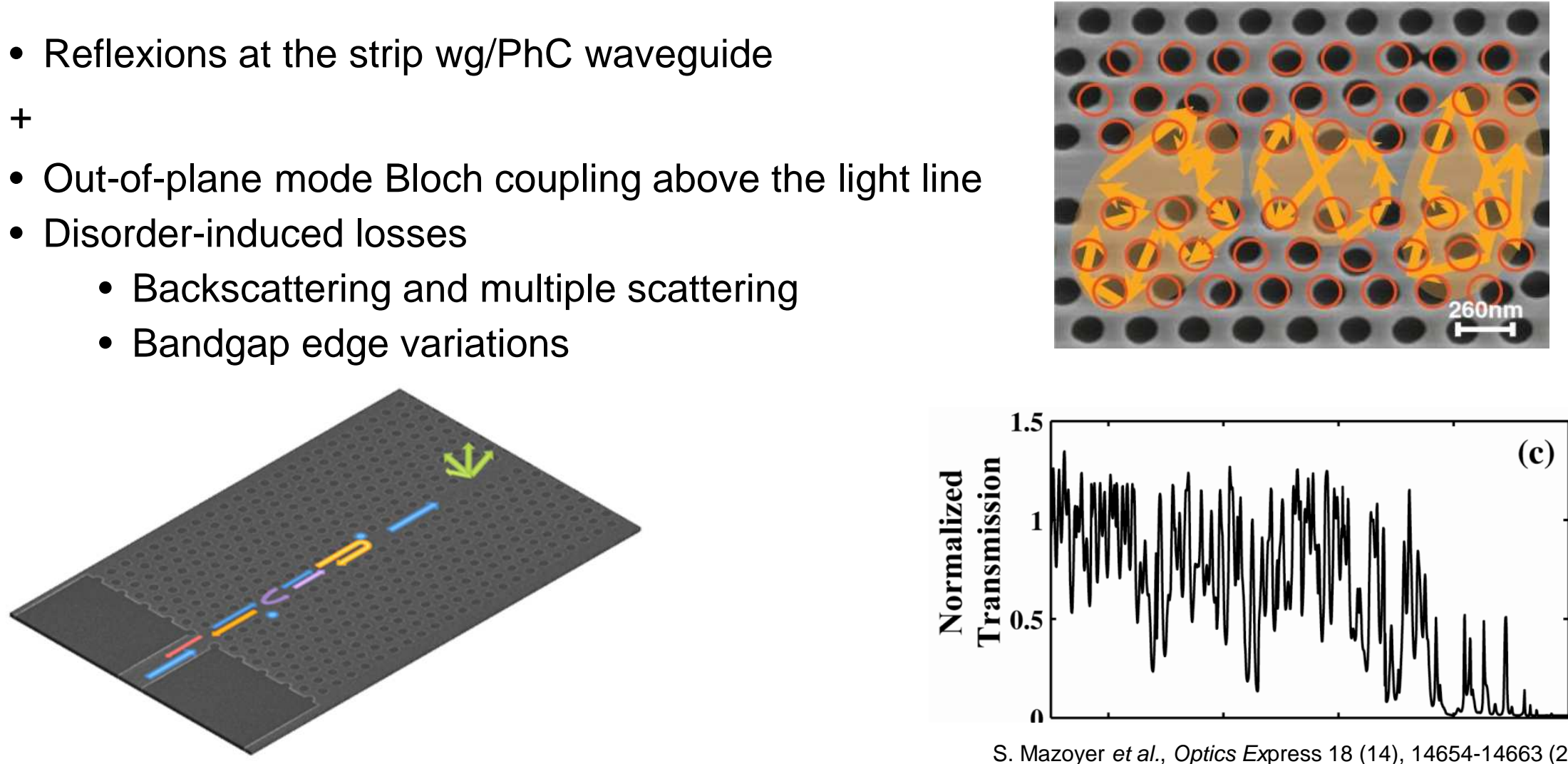

S. Mazoyer et al., Optics Express 18 (14), 14654-14663 (2010).

=> The issue of minimizing losses in slotted PhC waveguides is of paramount importance for applications 


\section{Slotted photonic crystal waveguides}

- Previous results: similar losses for fast waves $\left(n_{G} \sim 2-5\right)$ as in $\mathrm{W} 1 \mathrm{PhC}$ waveguides

- No results for slow waves

\section{Our recent works:}

- Design of slotted PhC waveguides engineered to exploit the W1-like mode

- Fabrication of the structures of different lengths from $50 \mu \mathrm{m}$ to $1 \mathrm{~mm}$

- Optical characterization by using a modified Optical Low Coherence Reflectometry (OLCR) technique entitled Optical Coherent Tomography (OCT):

- Measurement of the propagation losses as a function of the light group index

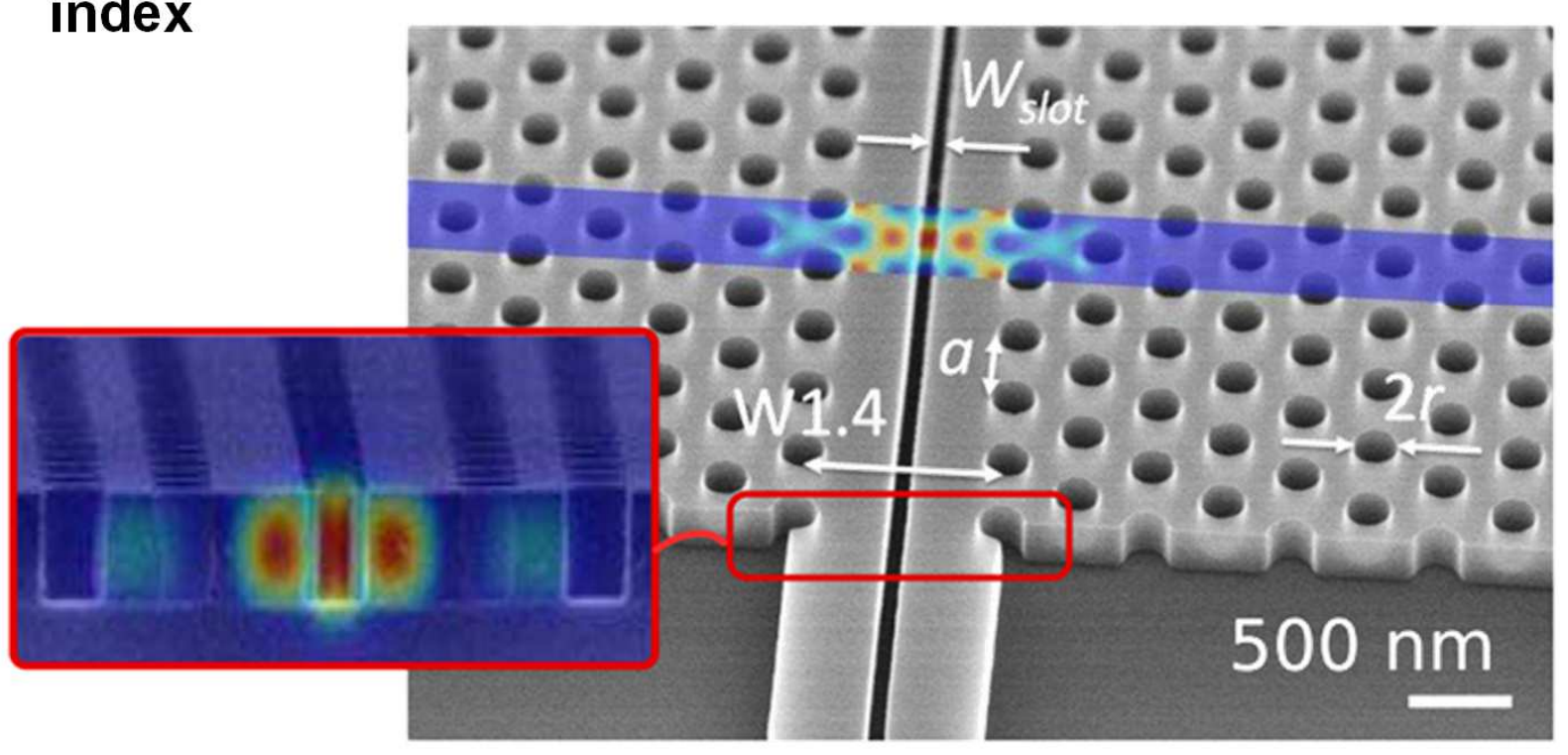




\section{Management of the light coupling issue}

Three steps are necessary:

1) Strip waveguide $=>$ slot waveguide

2) Slot waveguide $=>$ Fast light PhC Slot waveguide

3) Fast light $\mathrm{PhC}$ Slot waveguide $=>$ Slow light $\mathrm{PhC}$ Slot waveguide
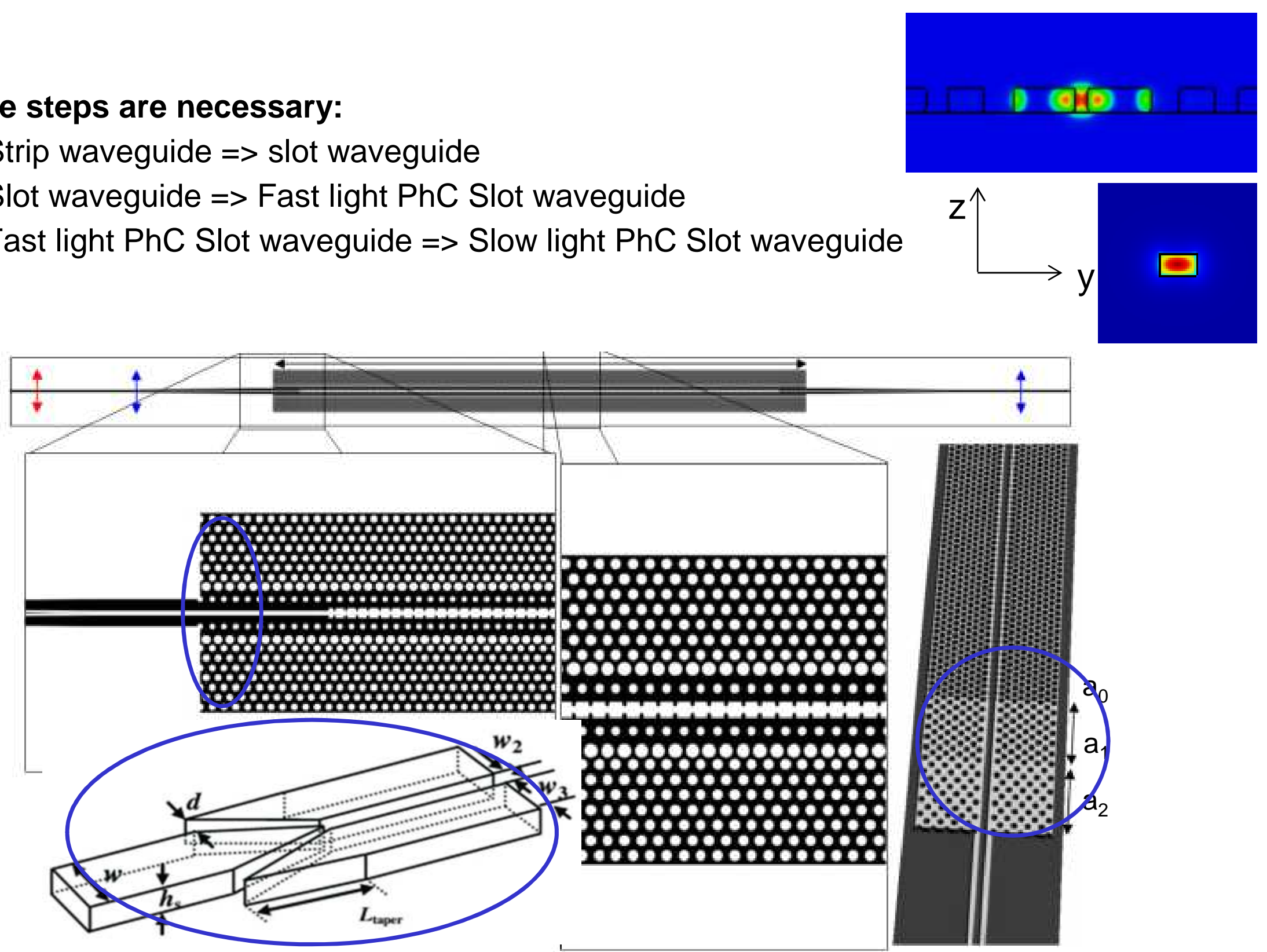

Z. Wang et al., Opt. Lett. 34 (10), 1498,(2009). 


\section{Fabrication and characterization of the slot photonic crystal waveguides}

- SOITEC 6" wafers of $260 \mathrm{~nm}$ Si film and $2 \mu \mathrm{m}$ BOX

- 80kV e-beam lithography Nanobeam NB-4 forming the pattern in a ZEP-520A resist mask

- ICP RIE etching of the silicon

- Removal of the resist by $\mathrm{O}_{2}$ plasma

- Cleavage of the sample

$+$
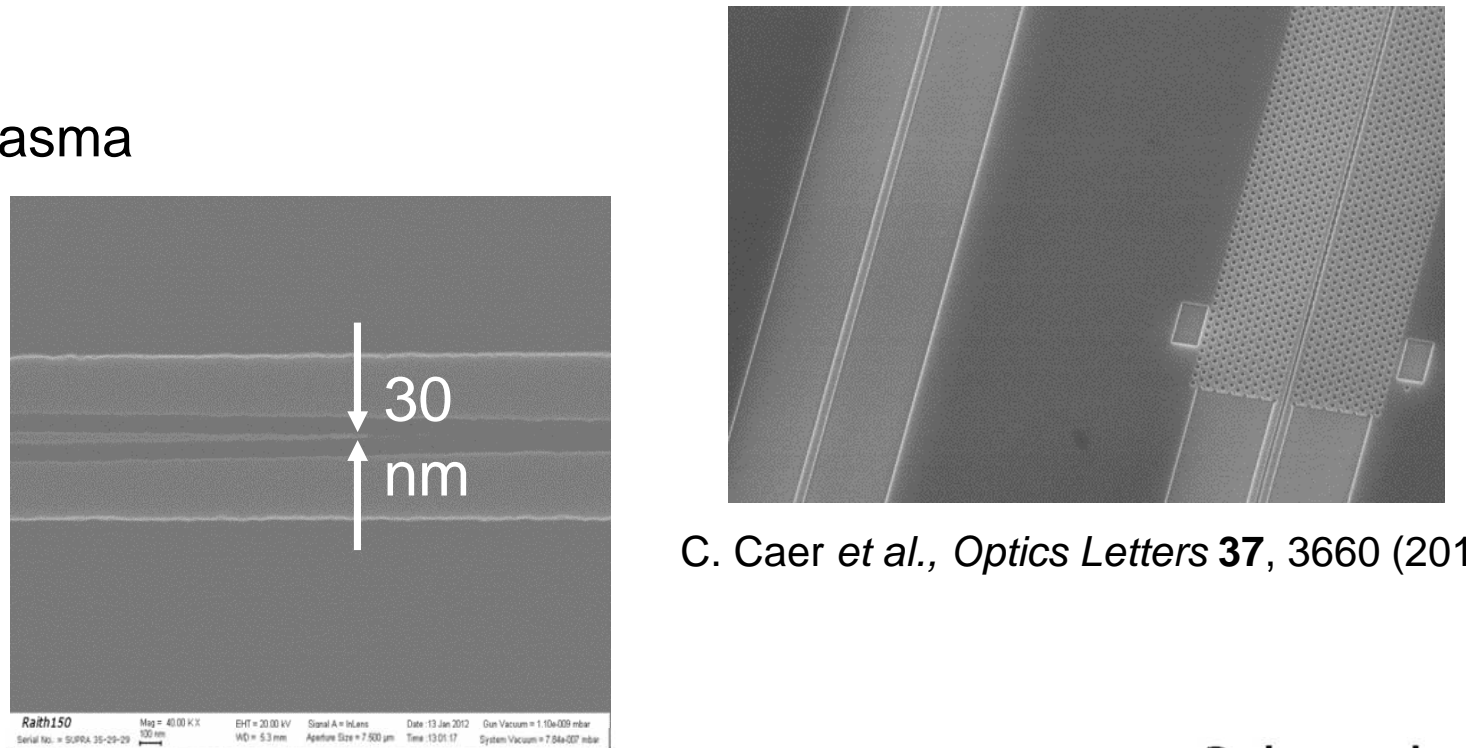

C. Caer et al., Optics Letters 37, 3660 (2012)

Optical Coherent Tomography (OCT)
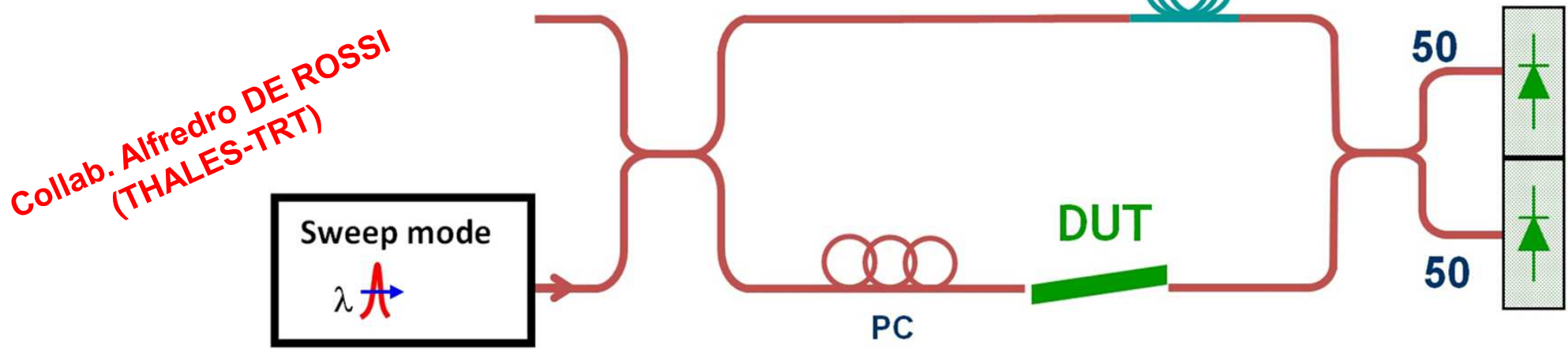

Balanced Photodetector

L

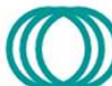




\section{$1 \mathrm{~mm}$-long slot photonic crystal waveguide}

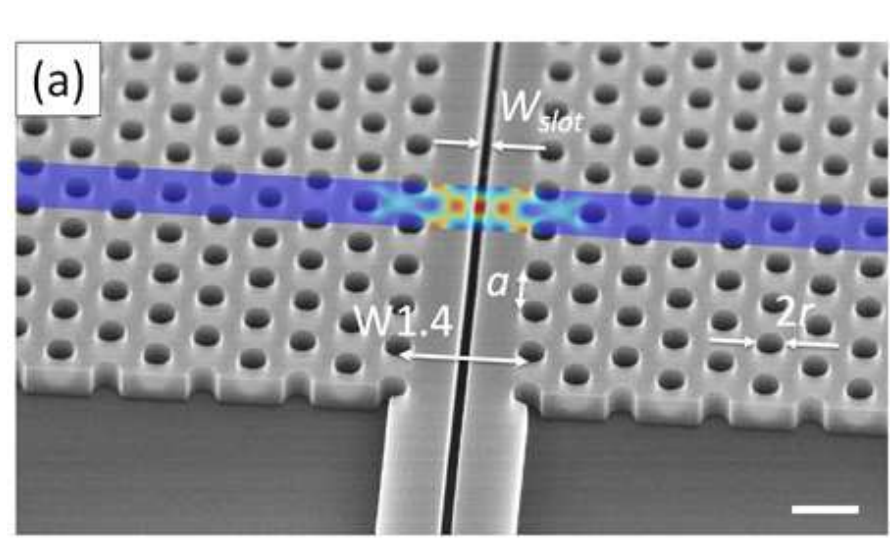

$a=400 \mathrm{~nm}, W 1.4=1.4 \sqrt{ } 3 a=970 \mathrm{~nm}$

- Mode partially confined in the slot

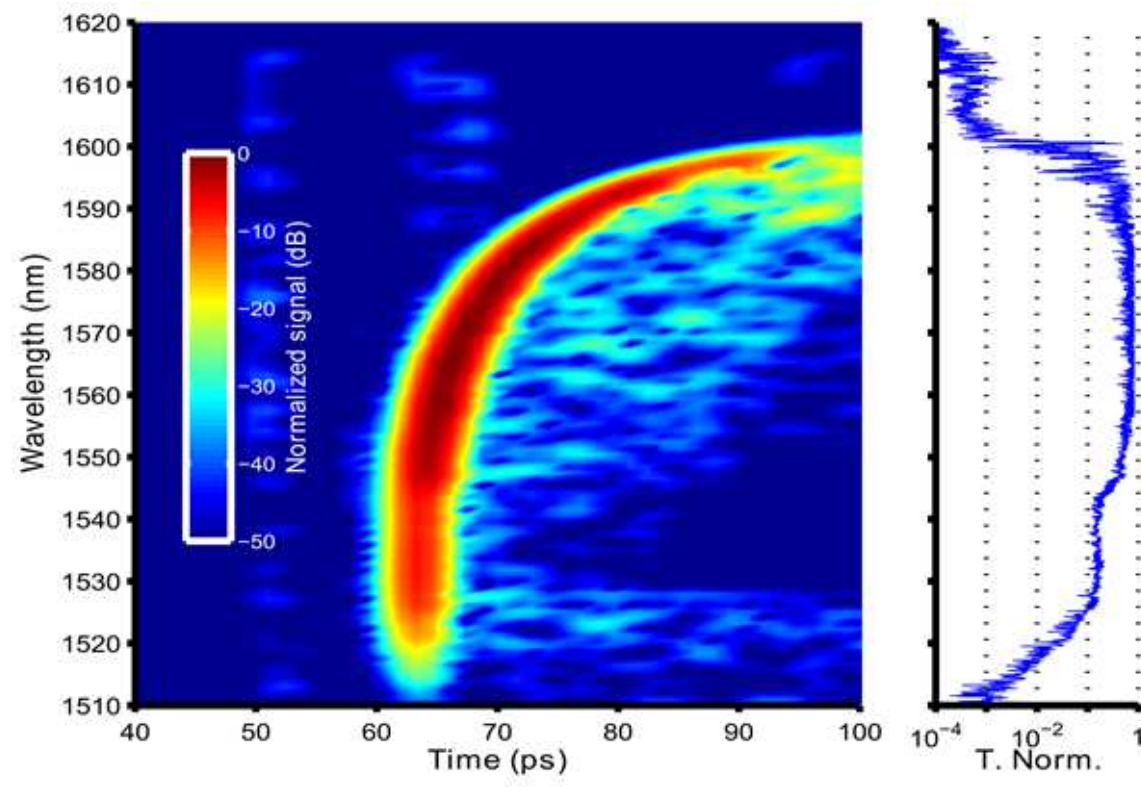

- Cut-off wavelength around $\lambda=1590 \mathrm{~nm}$

- Delay ranging from 60ps to $100 p s$ for $n_{G}$ in the 5-21 range $r=105 \mathrm{~nm}, W_{\text {slot }}=100 \mathrm{~nm}$

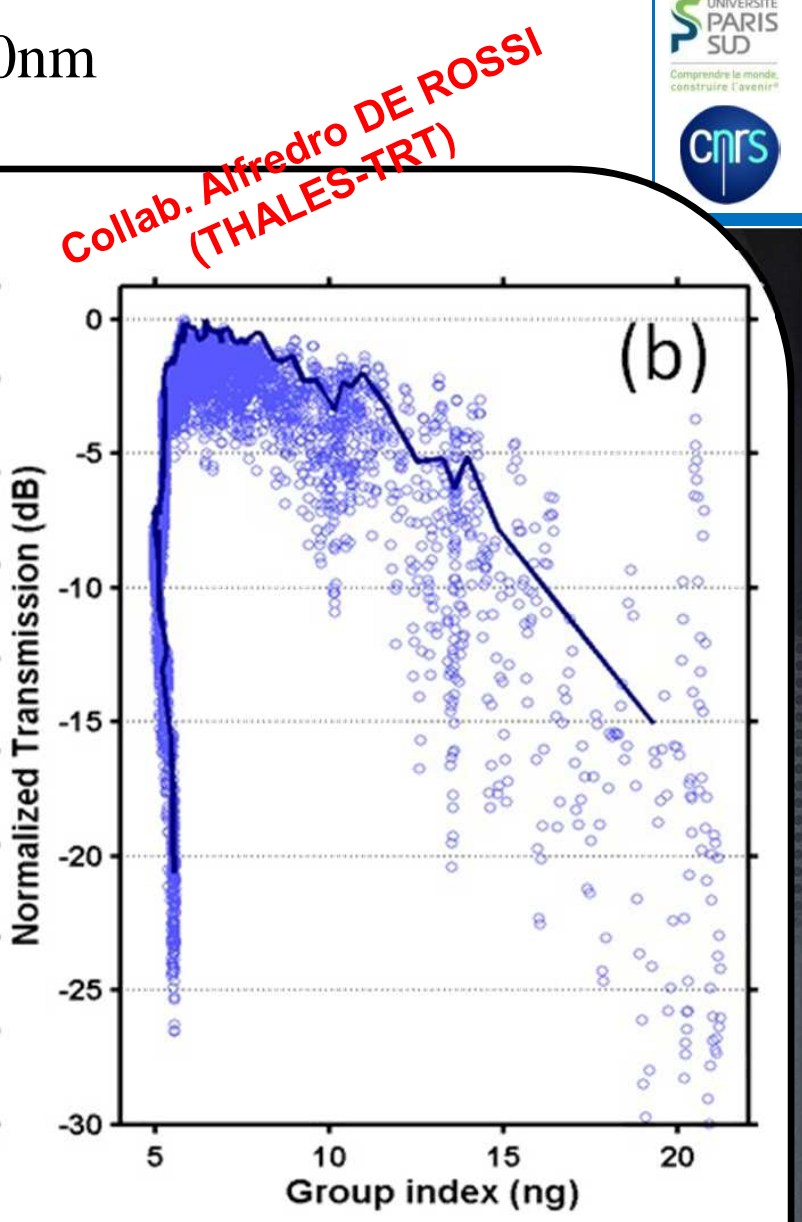

- Good agreement of experimental dispersion results with 3D-PWE calculation

- Low loss for small $n_{G}$ value, even for this long photonic crystal waveguide

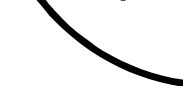




\section{Variable slot photonic crystal waveguides: $50 \mu \mathrm{m}=>1 \mathrm{~mm}$}

Collab. Alfredro DE ROSSI
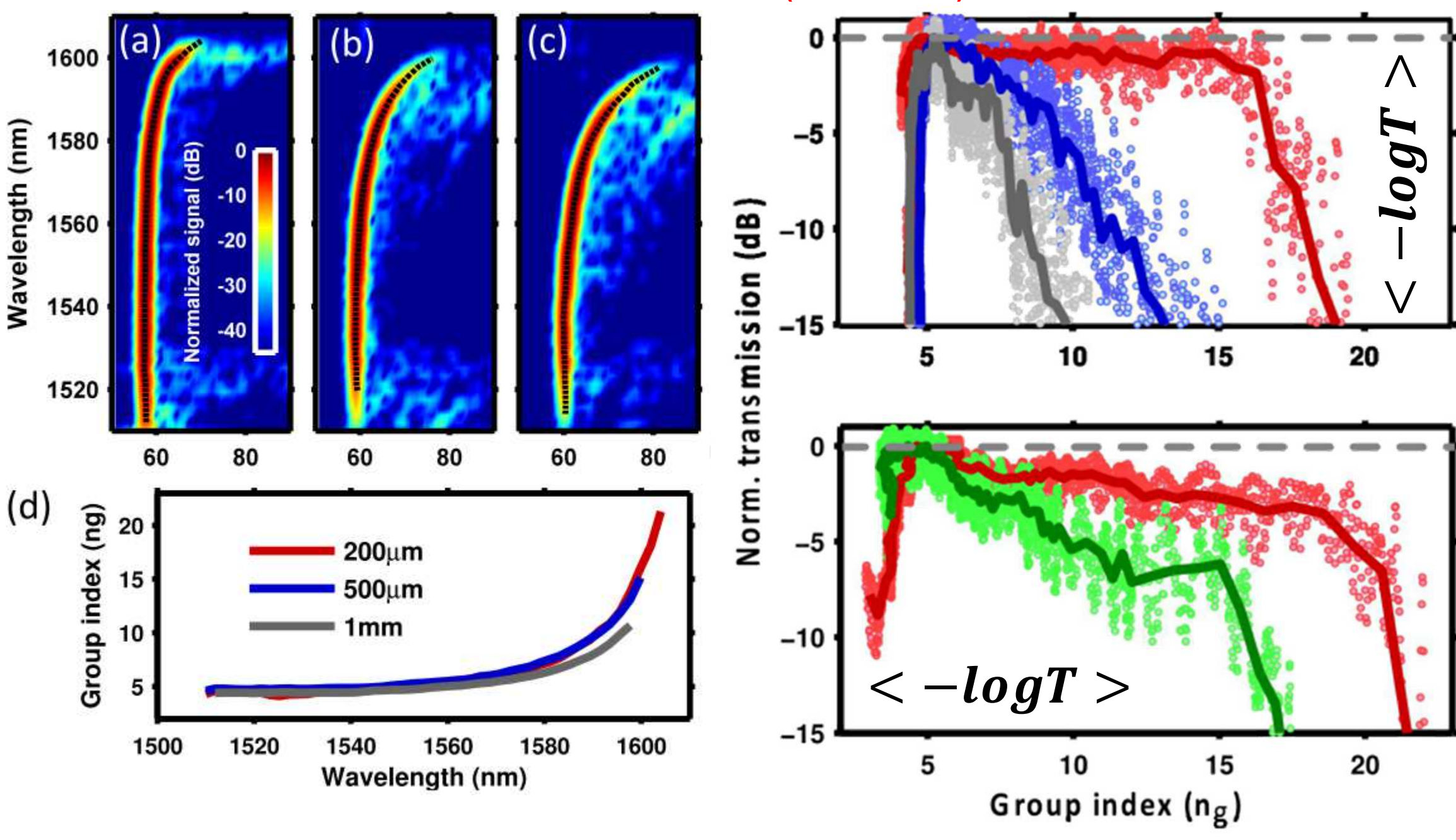

Good reproductibility of the fabrication \& consistency of the fabrication-andcharacterization cycle

- Moderate losses for $L=200 \mu m$

- E-beam writing field: $300 \mu \mathrm{m}$ $=>$ influence of the stitching errors 


\section{Potentials for light-matter interactions}

Let $A_{\text {eff }}$ be defined as: $P=0.5\left|\mathbf{E}_{\text {slot }}\right|^{2} \eta^{-1} A_{\text {eff }}$

$A_{\text {eff }}=\frac{A_{\text {slot }}}{\phi} \frac{n_{\text {slot }}}{n_{g}} \quad$ with $\quad \phi=\frac{\int_{\text {slot,a }} \epsilon_{r}|\mathbf{e}|^{2} d S d x}{\int_{S, a} \epsilon_{r}|\mathbf{e}|^{2} d S d x}$

$$
\mathrm{FOM}=\frac{n_{\mathrm{g}}}{n_{\text {slot }}} \phi
$$

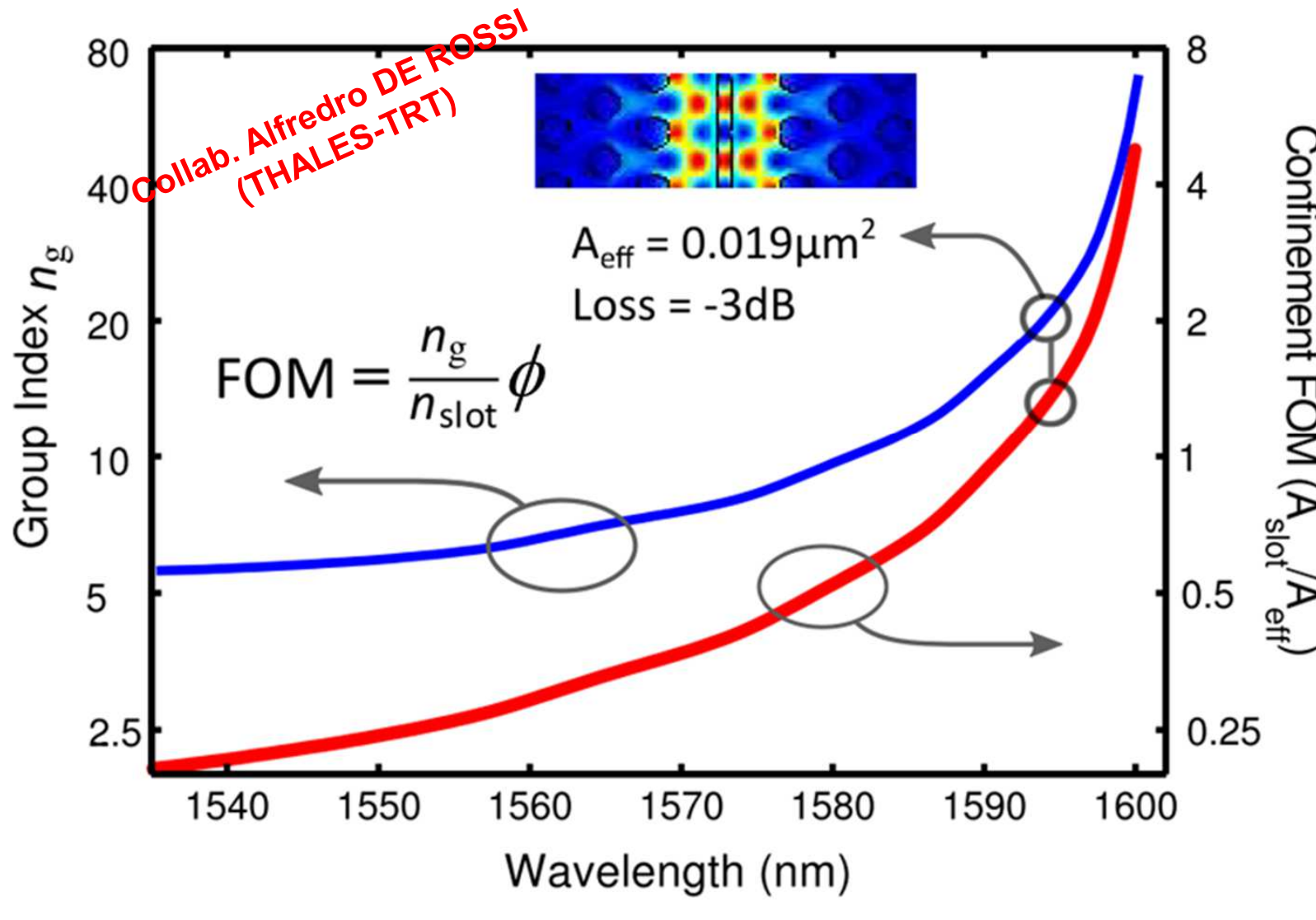

Increase of $n_{G}$ from 6 to 20

$=>A_{\text {eff }}$ is reduced by a factor of 5 down to around $0.02 \mu \mathrm{m}^{2}$ (down to 10 times lower than the diffraction limit) 


\section{Slotted Photonic Crystals: a great opportunity}

for the design of ultra-small resonators

\section{De $\circ$ 앙}

\section{D}

- 


\section{Slot Photonic Crystal cavities: the state-of-the art (1)}

August 1, 2010 / Vol. 35, No. 15 / OPTICS LETTERS 2523

Refractive index sensing with an air-slot photonic crystal nanocavity

Jana Jágerská, ${ }^{*}$ Hua Zhang, Zhaolu Diao, Nicolas Le Thomas, and Romuald Houdré
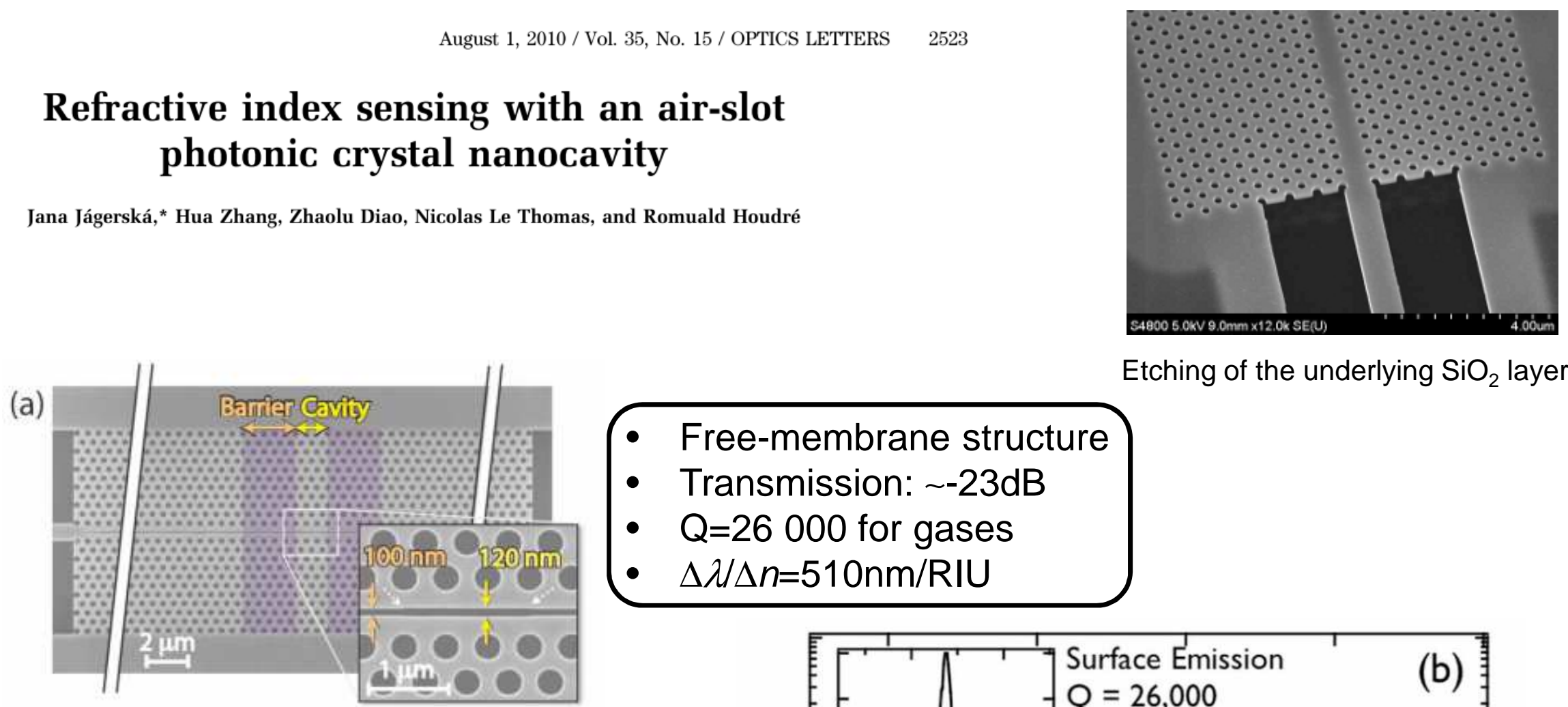

Etching of the underlying $\mathrm{SiO}_{2}$ layer

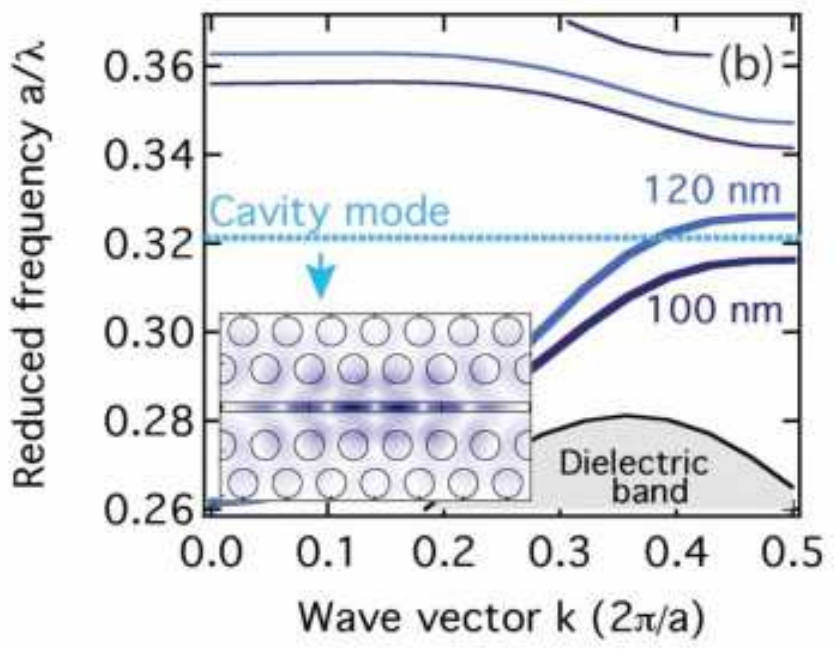

- Free-membrane structure

- Transmission: -23dB

- $\mathrm{Q}=26000$ for gases

- $\Delta \lambda / \Delta n=510 \mathrm{~nm} / \mathrm{RIU}$

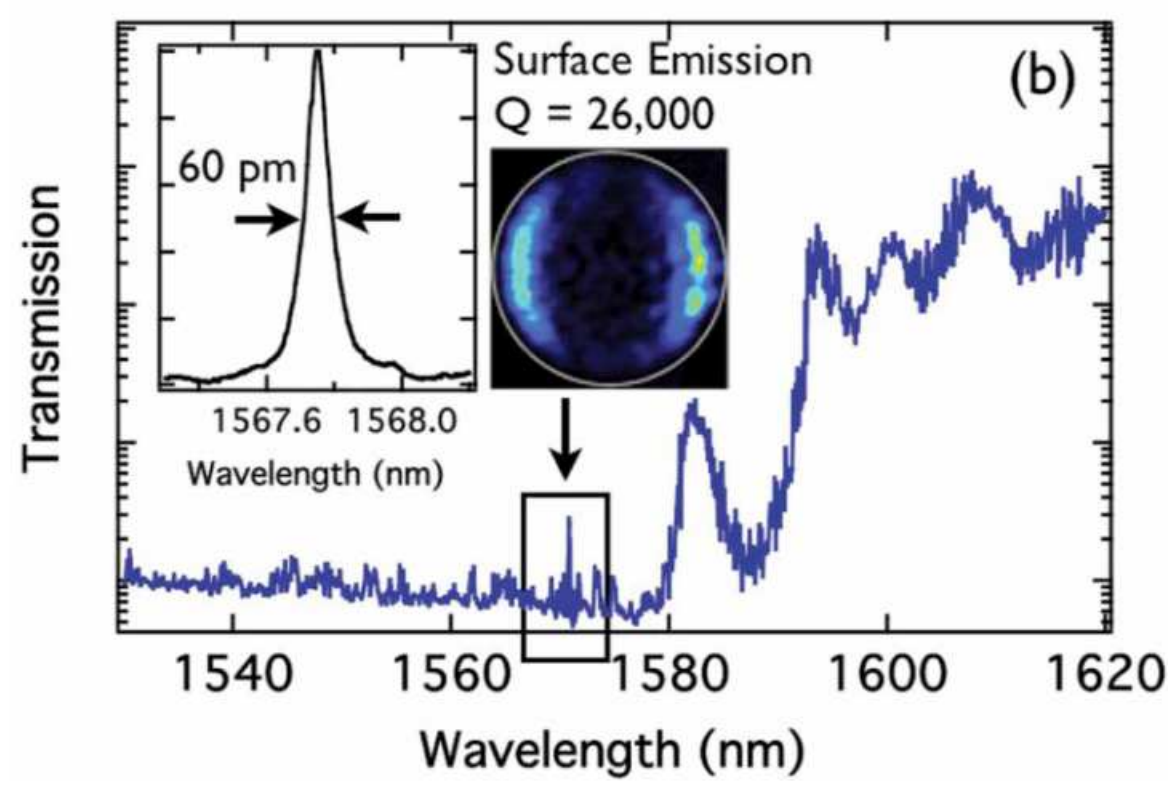




\section{Slot Photonic Crystal cavities: the state-of-the art (2)}

Sensors 2013, 13, 3675-3710; doi:10.3390/s130303675

Mark G. Scullion *, Thomas F. Krauss and Andrea Di Falco
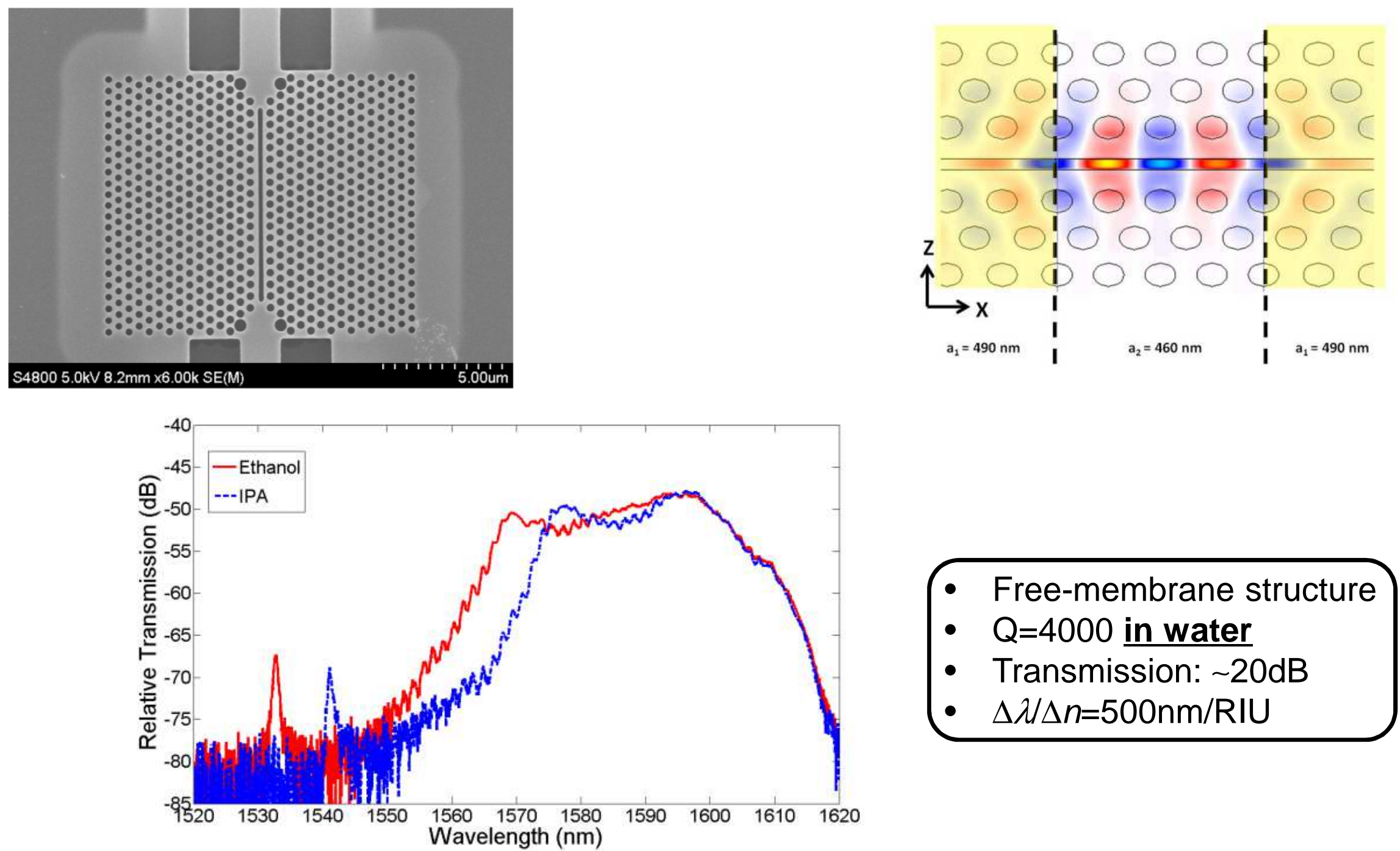


\section{SPCW cavities: the design approach (1)}
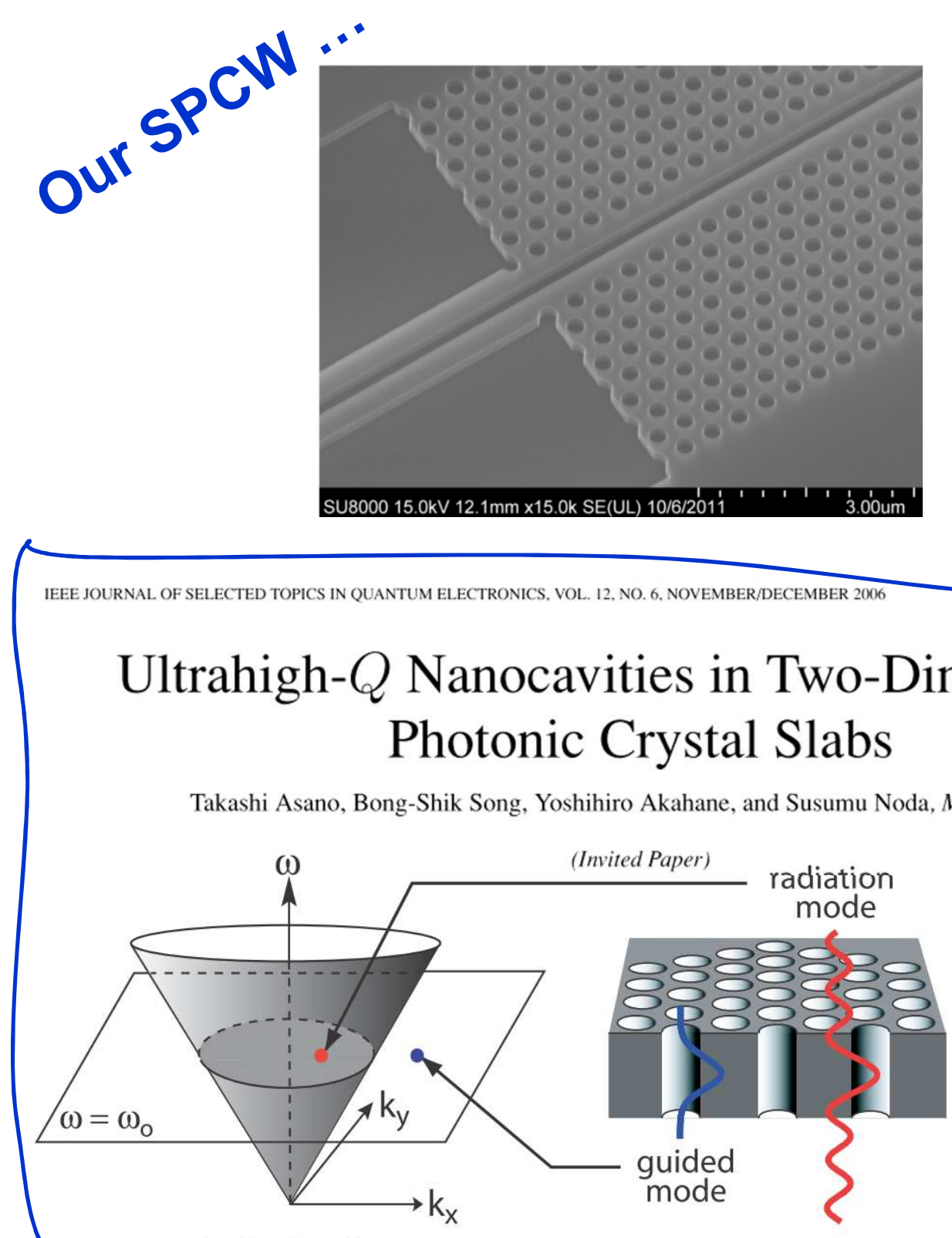

cone in $\left(k_{x}, k_{y}, \omega\right)$-space
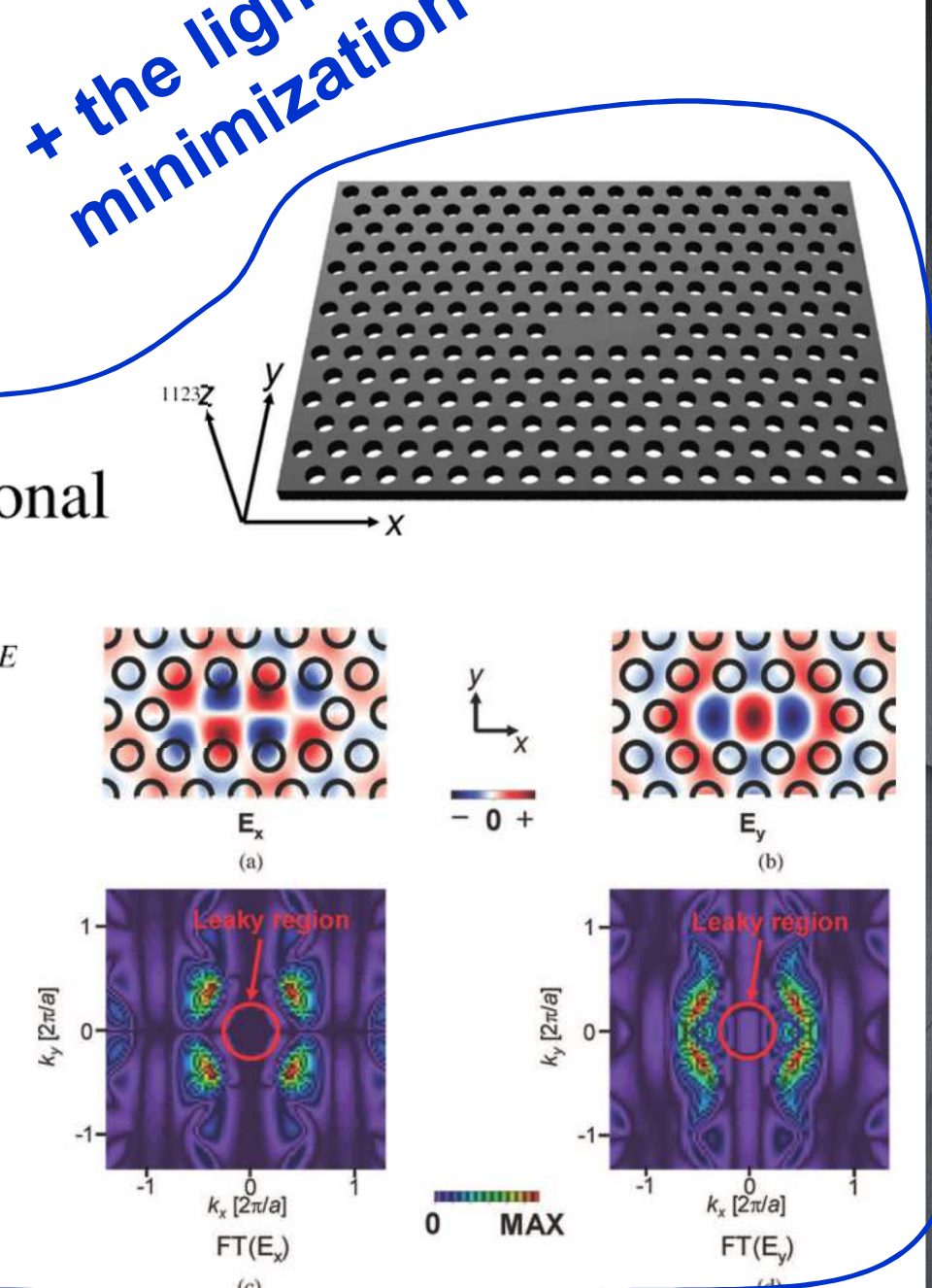


\section{SPCW cavities: the design approach (2)}

- Starting slot photonic crystal waveguide: $a_{0}=400 \mathrm{~nm}$ and $W_{\text {slot }}=100 \mathrm{~nm}$

- Double-step heterostructure by elongating the lattice constant in the propagation direction to $a_{1}=410 \mathrm{~nm}$ and then $\mathbf{a}_{2}=\mathbf{4 2 0} \mathrm{nm}$, respectively.
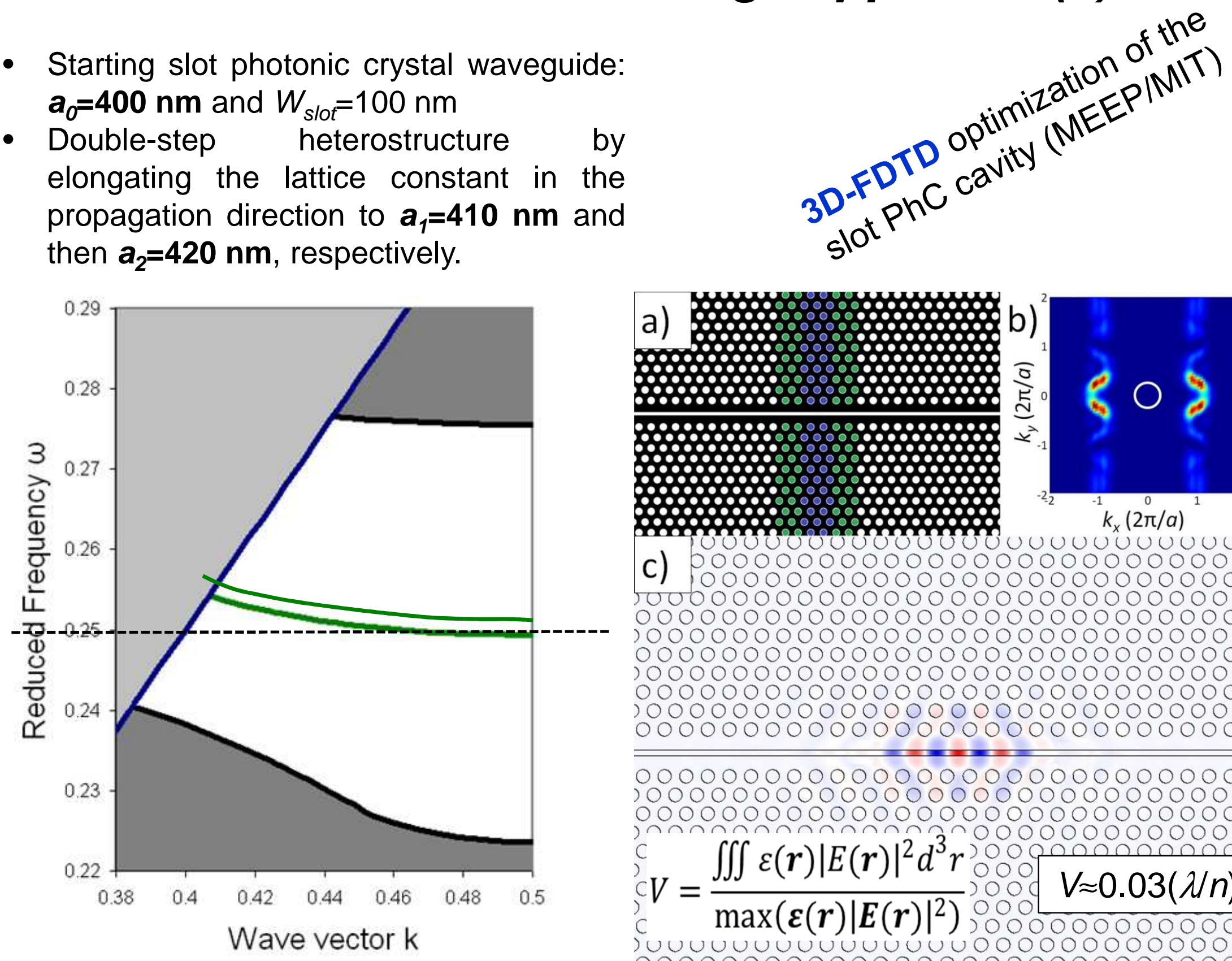

a)
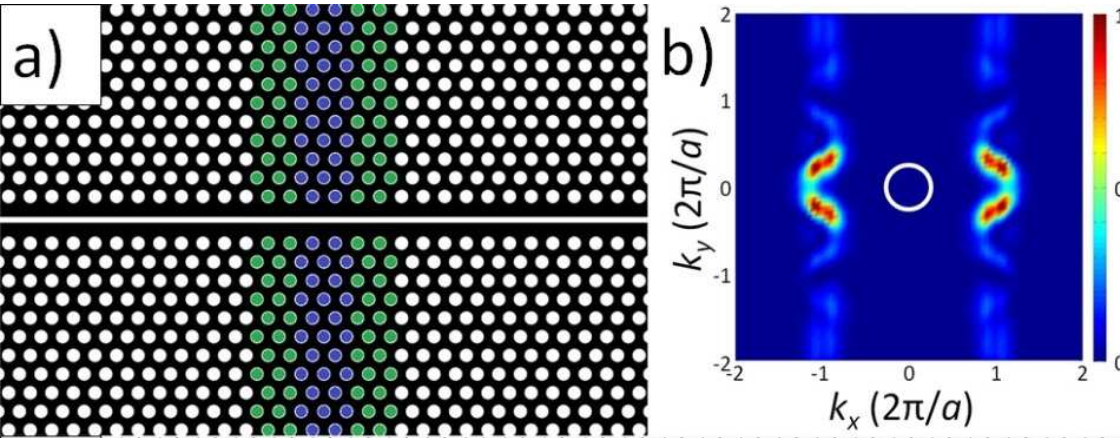

$k_{x}(2 \pi / a)$

c) (4) 200000000000000000000000000000 000000000000000000000000000000 00000000000000000000000000000

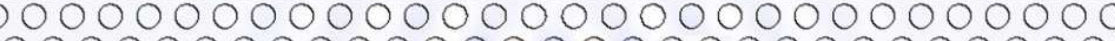
000000000000000000000000000000

000000000000000000000000000000 00000000000000000000000000000 o.

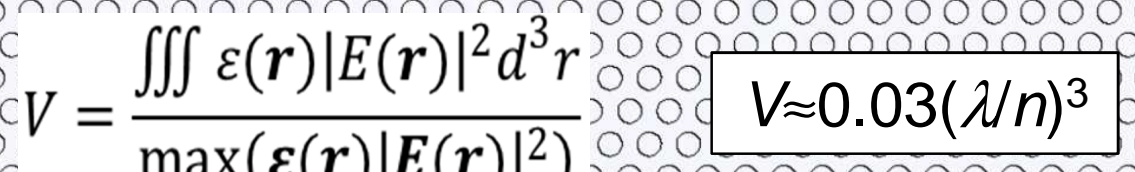
$\max \left(\boldsymbol{\varepsilon}(\boldsymbol{r})|\boldsymbol{E}(\boldsymbol{r})|^{2}\right), 000000000000000$

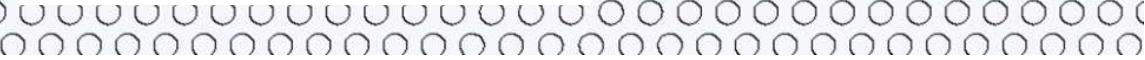




\section{Characterization setup}

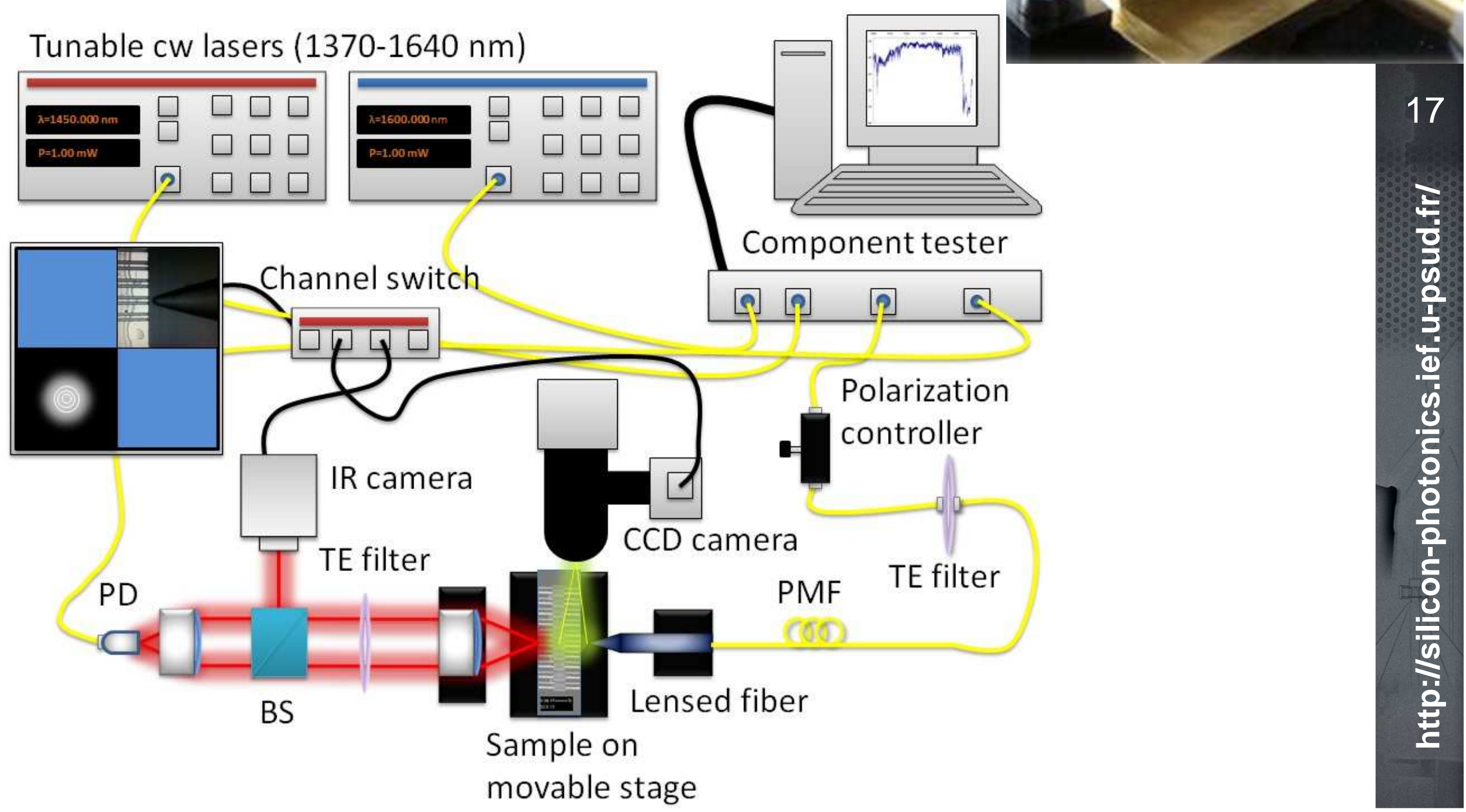




\section{Filled slot PhC cavity: experimental results}

Filling by a 1.46 index liquid.
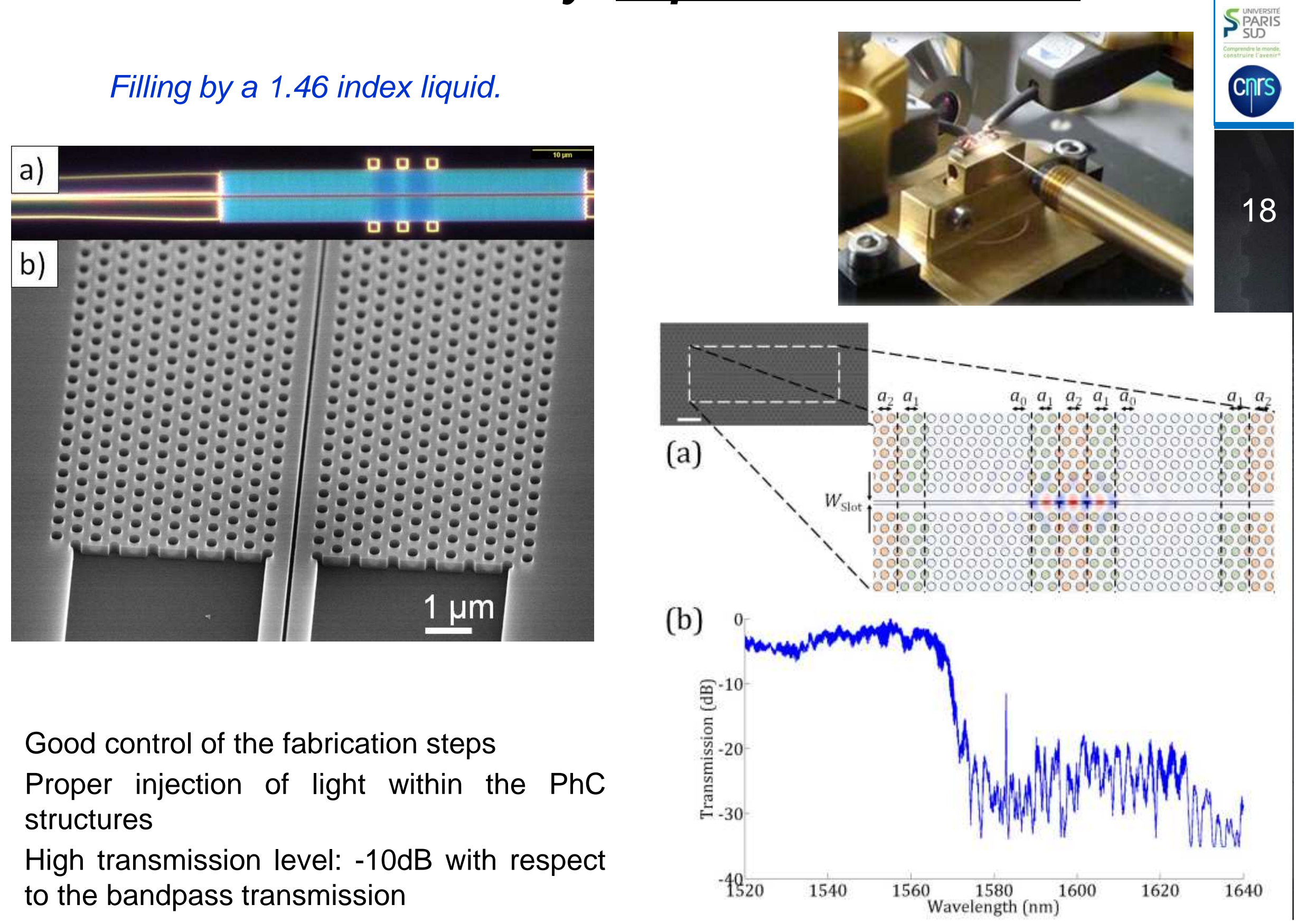

- Good control of the fabrication steps

- Proper injection of light within the PhC structures

- High transmission level: $-10 \mathrm{~dB}$ with respect to the bandpass transmission

(b) 


\section{Filled slot PhC cavity: experimental results}

- Measurements performed after covering the PhC structure by a Cargille liquid index ranging from $\sim 1.35$ to $1.55: 1.345,1.41,1.448,1.516$, and 1.545 .

- Normalization of the spectra by the input laser diode power ( $P i n=5 \mathrm{~mW}$ )

- NO FILTERING APPLIED TO THE DATA

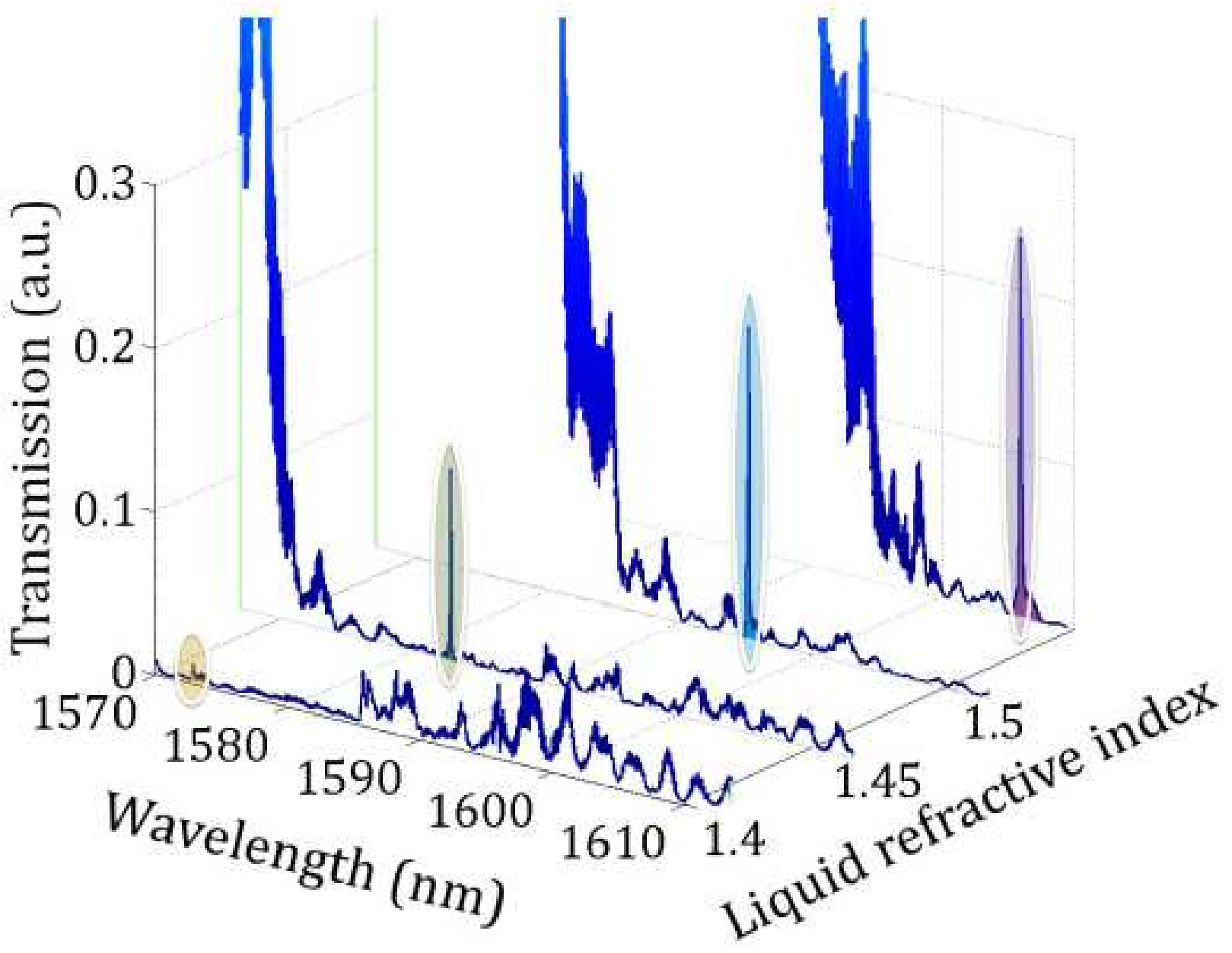




\section{Filled slot PhC cavity: experimental results}
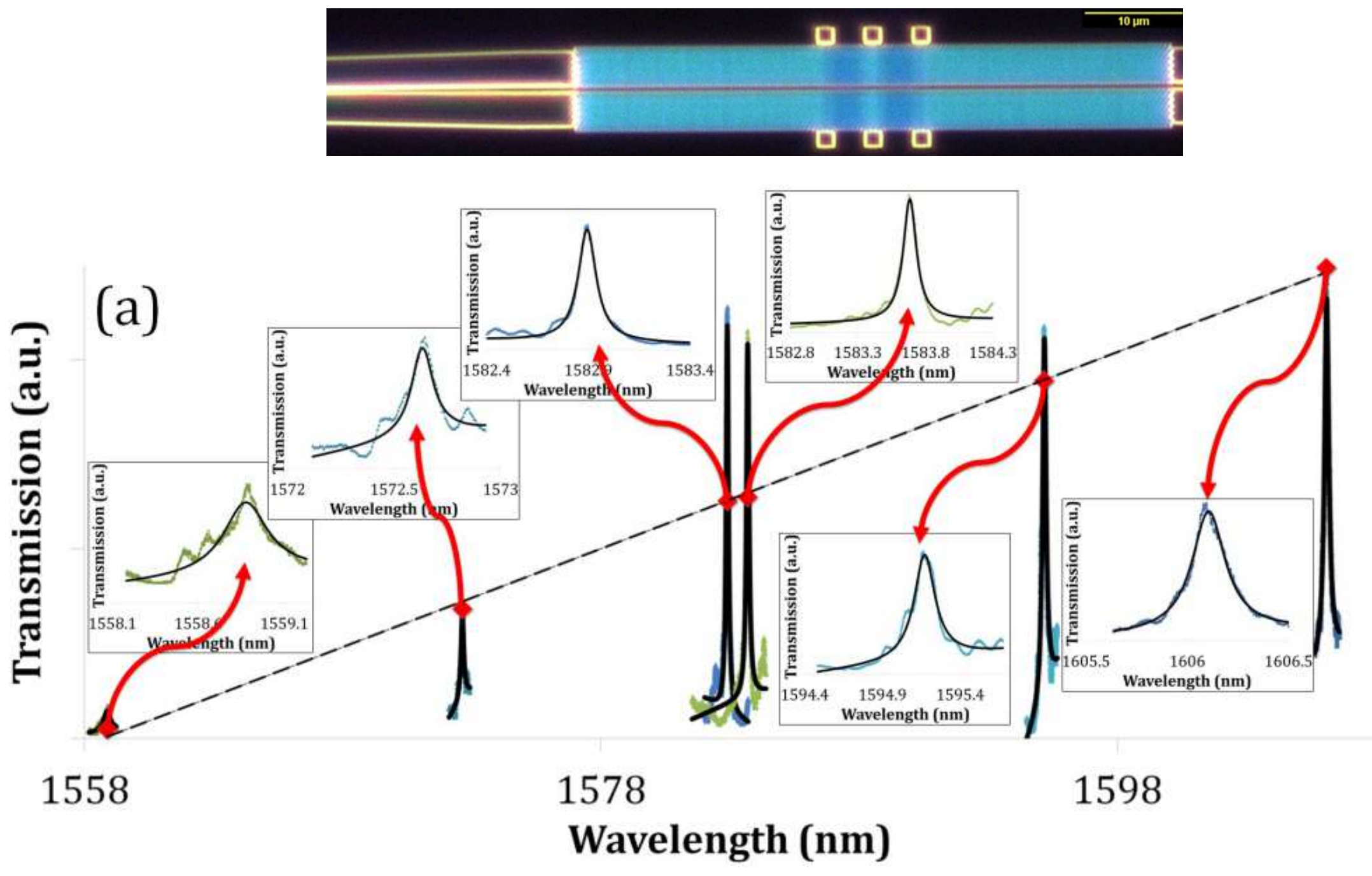

1.545

Wavelength (nm)

- Well identified resonance peaks for the different index values.

- The spectrum asymmetry slightly increases with the vertical index mismatch 
Filled slot PhC cavity: experimental results

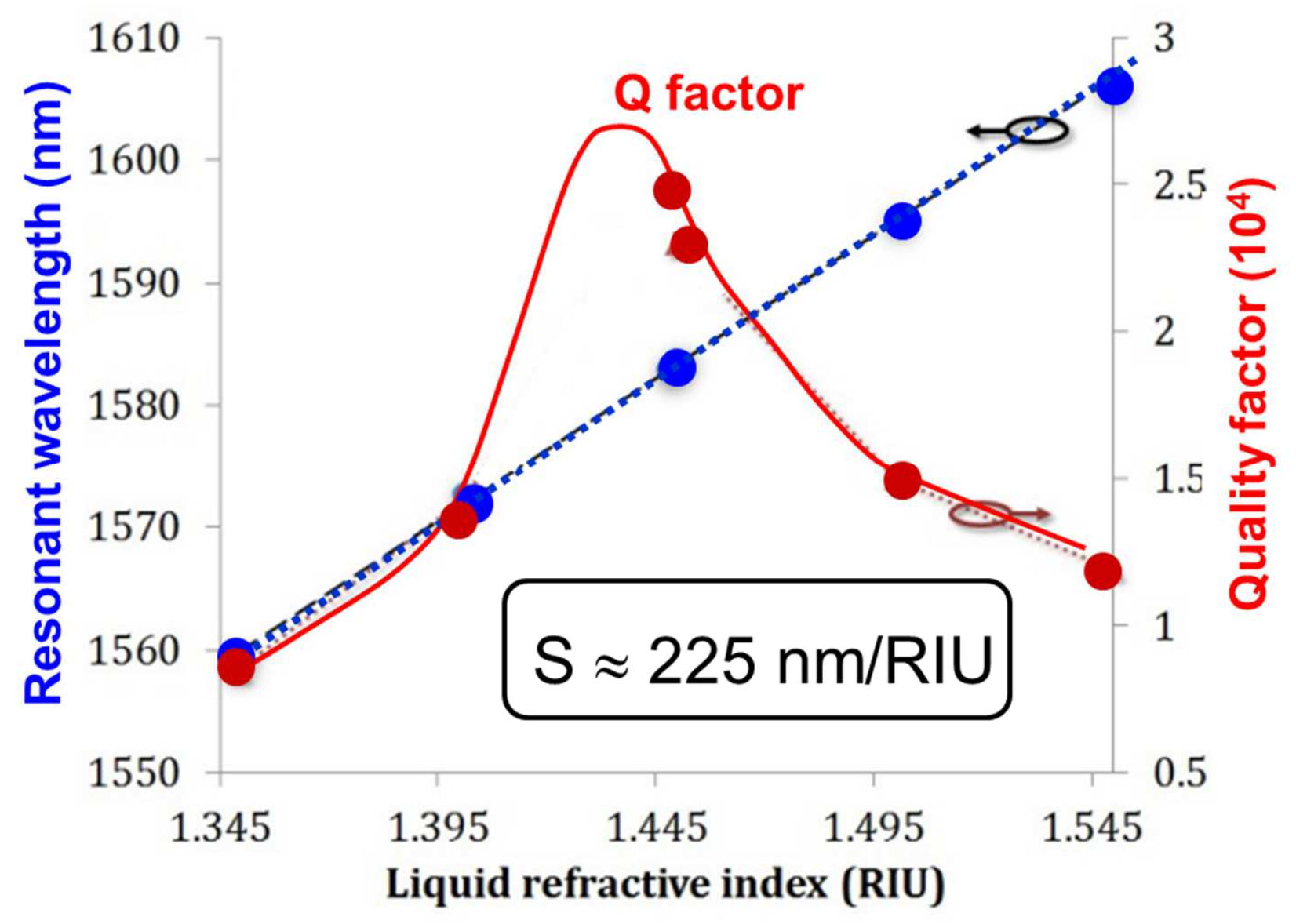

- Quality factors up to 26,000 for non-freestanding slotted photonic crystal cavities filled by liquids ensuring a good mechanical stability.

- Silicon photonics process (SOI wafers) 


\section{Conclusion}

- Slow light slot photonic crystal waveguides:

o Investigation of the slot $\mathrm{PhC}$ waveguide dispersion curve by direct group-velocity-time-of-flight optical measurements (OCT)

- Experimental demonstration of low losses for group index values up to $n_{G}=20$

○ Non-freestanding silicon slot $\mathrm{PhC}$ cavities infiltrated by liquids:

o With Q-factors 26,000

○ $V \approx 0.03(\lambda / n)^{3}=>Q / V \approx 700,000$

o Intrinsic Q-factor is above 200,000

o Transmission on resonance is $20 \%$ in the best cases

o Sensitivity of devices around $225 \mathrm{~nm} / \mathrm{RIU}$

$\Rightarrow$ Biosensing and/or non linear optics by filling the slot with organic materials 


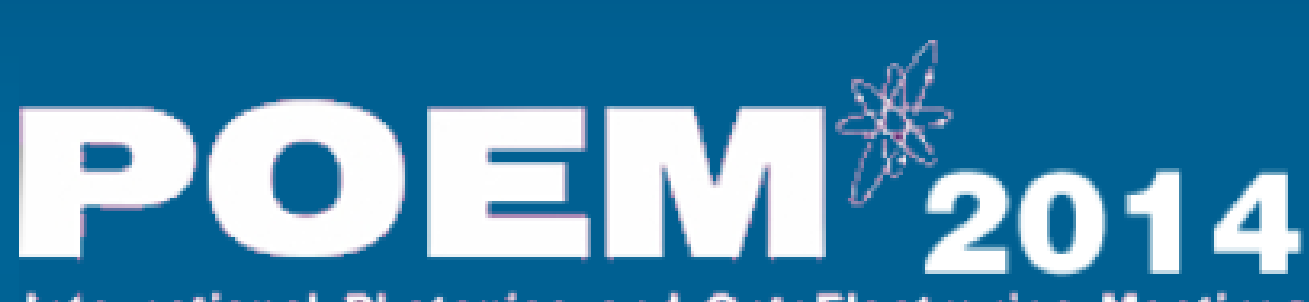

\section{Thank you}

for your attention

Eric CASSAN

eric.cassan@u-psud.fr 